\title{
Inländische Wanderungen in Deutschland - wer gewinnt und wer verliert?
}

\author{
Roland Busch
}

Eingegangen: 22. Oktober 2015 / Angenommen: 15. Juli 2016 / Online publiziert: 30. August 2016 (C) Der/die Autor(en) 2016. Dieser Artikel ist eine Open-Access-Publikation.

Zusammenfassung Die derzeitige Debatte um die Zukunftsfähigkeit der Städte ist sehr stark von der Zuwanderung aus dem Ausland dominiert. Durch diese werden inländische Wanderungstrends derzeit jedoch ,,überdeckt“, was zu Fehlinterpretationen bei der Betrachtung der kommunalen Wanderungssaldi führen kann. In der folgenden Untersuchung wird das aktuelle Phänomen der Auslandszuwanderung deshalb ausgeblendet und der Fokus auf die inländischen Wanderungstrends gerichtet.

Bei isolierter Betrachtung der inländischen Wanderungsbewegungen zeigt sich, dass sich die Wanderungssaldi der Großstädte in den letzten Jahren negativ entwickelt haben. Allerdings gibt es große regionale Unterschiede: Während viele prosperierende Großstädte zwischen 2008 und 2014 einen starken Rückgang des inländischen Wanderungssaldos zu verzeichnen hatten und einige in den letzten Jahren sogar Einwohner an die Kreise verloren, sind in der Region Mitteldeutschland und in Teilbereichen Nordrhein-Westfalens davon abweichende Entwicklungen festzustellen. Dies deutet auf ökonomisch bedingte Verdrängungsprozesse in Großstädten mit stark angespanntem Wohnungsmarkt hin. Anscheinend wird es insbesondere für Haushalte in der Eigentumsbildungs- und Familiengründungsphase in Großstädten mit stark steigenden Miet- und Kaufpreisen immer schwieriger, sich die Wohnwünsche zu erfüllen und der Umzug in das preiswertere Umland gewinnt als Alternative an Bedeutung. Dass Großstädte aber immer noch eine hohe Attraktivität als Wohnstandorte haben, zeigen die weiterhin starken inländischen Wanderungsgewinne derjenigen Großstädte, die (derzeit noch) über relativ entspannte Wohnungsmärkte verfügen. Es ist davon auszugehen, dass sich bei einem Rückgang der Auslandszuwanderung der Druck auf die großstädtischen Immobilienmärkte etwas reduziert, was negative Auswirkungen vor allem auf die aktuellen Gewinner der

R. Busch (四)

Bergische Universitat Wuppertal, Wuppertal, Deutschland

E-Mail: rbusch@uni-wuppertal.de 
Verdrängungsprozesse - die suburbanen und ländlichen Kommunen im Umfeld der Großstädte - haben könnte.

Schlüsselwörter Bevölkerungsentwicklung · Wanderungsbewegungen · Binnenwanderung · Suburbanisierung · Attraktivität von Wohnimmobilienmärkten · Nachhaltige Investitionsstandorte

\section{Domestic migration in Germany - who are the winners and losers?}

Abstract The current debate on urban sustainability is dominated by the strong foreign migration. In the last years this migration "overshadowed" the domestic migration trends, which can lead to misinterpretation when analyzing migration balance data. Therefore the following analysis fades out the current phenomenon of foreign immigration and focuses on domestic migration trends.

When doing this, the statistics shows us that the domestic migration balances of the big cities have declined markedly in recent years. Albeit there are large regional differences: while a deterioration in the domestic migration balances took place in many fast growing cities between 2008-2013 and some of these cities even lost residents to the surrounding municipalities in recent years, an opposite development can be observed in the region of Central Germany and in parts of North Rhine-Westphalia. This indicates economically-induced displacement processes in large cities with a tensed housing market. It seems to get more and more difficult apparently for households which are thinking about an acquisition of house property or about starting a family to satisfy their housing requirements in cities with a tensed housing market - so they are forced to relocate in regions with lower house prices. On the other hand the continued strong domestic migration gains of those big cities, with (still) have relatively relaxed housing markets, shows the sustained attractiveness of city-life for many people. It is assumed, that the pressure on the metropolitan real estate markets will diminish, when foreign immigration will decline. This could have negative effects especially for the current winners of the displacement processes the suburban and rural municipalities surrounding the large cities.

Keywords Population development - migration flows · Domestic migration · Suburbanization - Deurbanisation - Attractiveness of regional housing markets · Sustainable investment locations

\section{Binnenwanderungssaldo als Indikator für die Raum- und Wohnungsmarktbeobachtung}

Wanderungen beeinflussen die Veränderungen der Bevölkerungszahl einer Region deutlich stärker als die natürlichen Bevölkerungsbewegungen, die sich aus den Geburten- und Sterbefällen ergeben (Birg 2013). Der Wanderungssaldo einer Kommune und seine Entwicklung geben wichtige Hinweise auf ihre Attraktivität und Zukunftsfähigkeit und sind als Indikatoren für die Untersuchung räumlicher Entwicklungsperspektiven sowie für die Wohnungsmarktbeobachtung von großer Bedeutung. So- 
wohl die Außen- als auch die Binnenwanderungen werden deshalb unter anderem für die Trendberechnungen der Raumordnungsprognosen und der Wohnungs- und Immobilienmarktbeobachtung des Bundesinstituts für Bau-, Stadt- und Raumforschung (BBSR) als wichtige Kennzahlen und Stellgrößen herangezogen (BBSR 2015, 2010; Milbert 2015).

Die zu beobachtende starke Auslandszuwanderung überdeckt aufgrund der staatlich gesteuerten Verteilung der Zuwanderer (Stichwort „Königsteiner Schlüssel“) und ihrer besonderen Standortpräferenzen derzeit jedoch die Aussagefähigkeit der allgemeinen Zahlen zur Wanderung auf kommunaler Ebene. Die Effekte der Auslandswanderung liegen wie ein „Schleier“ über der tatsächlichen Binnenentwicklung und können bei Untersuchungen zur Attraktivität und Zukunftsfähigkeit von Standorten zu falschen Schlüssen führen. Aus diesem Grunde soll in der folgenden Untersuchung durch die isolierte Betrachtung der Binnenwanderungssaldi der Blick darauf gerichtet werden, wie die Kommunen im innerdeutschen Wettbewerb um die Einwohner positioniert sind.

Die alleinige Betrachtung der Binnenwanderung erfolgte bisher meist im Rahmen spezieller Fragestellungen wie der Untersuchung der Stadt-Umland-Wanderung (Münter 2012; Hirschle und Schürt 2008), des Wanderungsverhaltens Hochqualifizierter (Deschermeier und Müller 2012), des alters- oder geschlechtsspezifischen Wanderungsverhaltens (Friedrich 2008; Milbert et al. 2013) oder der Entwicklung von Teilräumen, z. B. Ostdeutschland (Ragnitz 2013). Ausführliche Auseinandersetzungen mit den Binnenwanderungsbewegungen in Deutschland in den 1990er und 2000er-Jahren erfolgten zudem von Schlömer (2009), Herfert und Osterhage (2012) sowie Sander (2014), wobei diese auch differenzierte Betrachtungen von Altersklassen und Wanderungsentfernungen umfassten. Es fehlen allerdings aktuelle Auswertungen zu den Binnenwanderungssaldi für die Kreise und kreisfreien Städte, die die Entwicklungen der letzten Jahre analysieren. Im Rahmen der folgenden Untersuchung wurden diese Auswertungen für die Jahre 2008-2014 durchgeführt mit dem Ziel, eine Einschätzung zur Attraktivität von Lebens- bzw. Wohnstandorten losgelöst von der temporären Verzerrung durch die Auslandszuwanderung geben zu können.

\section{Methodik der Untersuchung}

Für die Untersuchung wurden die Daten der amtlichen Wanderungsstatistik (Statistik der räumlichen Bevölkerungsbewegung) aus den Jahren 2008 bis 2014 ausgewertet. Aus den von den Statistischen Ämtern des Bundes und der Länder bereitgestellten allgemeinen Angaben zu den jährlichen $\mathrm{Zu}$ - und Fortzügen in den Kreisen und kreisfreien Städte wurden die auslandsbezogenen $\mathrm{Zu}$ - und Fortzüge ausgeklammert und so die Binnenwanderungssaldi ermittelt. Die Entwicklung der Auslandswanderung und der inländischen Wanderungsbewegungen können so separat betrachtet werden. Für die Zeitreihenuntersuchung wurden die Kreise und kreisfreien Städten den vom BBSR im Rahmen der laufenden Raumbeobachtung verwendeten siedlungsstrukturellen Gebietstypen zugeordnet. Zur besseren Gegenüberstellung der Veränderungen 
in den unterschiedlich stark besetzten Gebietstypen wurden die Wanderungssaldi in Relation zur Einwohnerzahl (Saldo je 1000 Einwohner) gestellt.

Neben der allgemeinen Betrachtung der inländischen $\mathrm{Zu}$ - und Fortzüge erfolgten Auswertungen differenziert nach Altersklassen. Zudem fand eine Unterscheidung von innerdeutschen Nah- und Fernwanderungsbewegungen statt. Hierfür wurden die Angaben zu den Wanderungsbewegungen zwischen den einzelnen Kreisen und kreisfreien Städte aus der Kreiswanderungsmatrix mit den Distanzdaten einer Entfernungsmatrix zusammengeführt und analysiert. Die Kreiswanderungsmatrix 2014 wurde von den Statistischen Ämtern des Bundes und der Länder bezogen. Die benötigte Entfernungsmatrix konnte mit Hilfe der Eurostat-NUTS-3-Geodaten (Shapefiles) durch Berechnung der Luftlinienentfernungen zwischen den Mittelpunkten der Gebietsabgrenzungspolygone aller deutschen Kreise und kreisfreien Städte ermittelt werden. Da keine einheitlichen Angaben zur entfernungsbezogenen Abgrenzung zwischen regionaler (Nah-)Wanderung und über- bzw. interregionaler (Fern-) Wanderung in der Literatur existieren (Siedentop et al. 2014; Dittrich-Wesbuer und Osterhage 2008; Betz 1988; Schlömer 2009), werden in dieser Untersuchung zwei gebräuchliche Entfernungsgrenzen (100 und $150 \mathrm{~km})$ parallel betrachtet.

$\mathrm{Zu}$ beachten ist, dass durch die Neugliederung der Kreise und kreisfreien Städte in Mecklenburg-Vorpommern im Rahmen der Kreisgebietsreform 2011 eine Anpassung der Daten aus der Zeit vor 2011 durchgeführt werden musste. Da in den meisten Fällen lediglich eine Zusammenlegung von Kreisen bzw. kreisfreien Städten und nur in Einzelfällen eine Zuordnung eines alten Kreisgebietes zu mehreren neuen Kreisgebieten erfolgte, halten sich die Ungenauigkeiten durch die Anpassung allerdings in engen, tolerierbaren Grenzen.

\section{Die Entwicklung der Wanderungssaldi in den Kommunen}

Seit einigen Jahren ist in vielen Kommunen, die zuvor über Schrumpfung klagten, ein Anstieg der Einwohnerzahlen festzustellen. Etliche Kreise und kreisfreie Städte, die noch vor wenigen Jahren mit Abwanderungstendenzen konfrontiert waren, registrieren derzeit wieder enorme Zuzüge neuer Einwohner (siehe Abb. 1). Probleme wie Wohnungsmarktengpässe und z. T. stark ansteigende Immobilienpreise sind dadurch in vielen Regionen so akut wie schon lange nicht mehr (BMUB 2015).

Betrachtet man die Entwicklung der jährlichen Wanderungssaldi pro 1000 Einwohner in den Kreisen und kreisfreien Städten, so erkennt man eine deutliche Verbesserung in allen Gebietstypen. Abb. 2 stellt den Wanderungssaldo pro 1000 Einwohner differenziert für verschiedene siedlungsstrukturelle Gebietstypen dar.

Seit 2011 und 2012 zeichnen sich auch die ländlichen Kreise mit Verdichtungsansätzen und die dünn besiedelten ländlichen Kreise wieder durch - über alle Kommunen dieser Typen gerechnete - positive Wanderungssaldi aus, die größten Zugewinne an Einwohnern sind jedoch nach wie vor in den Großstädten zu verzeichnen. 

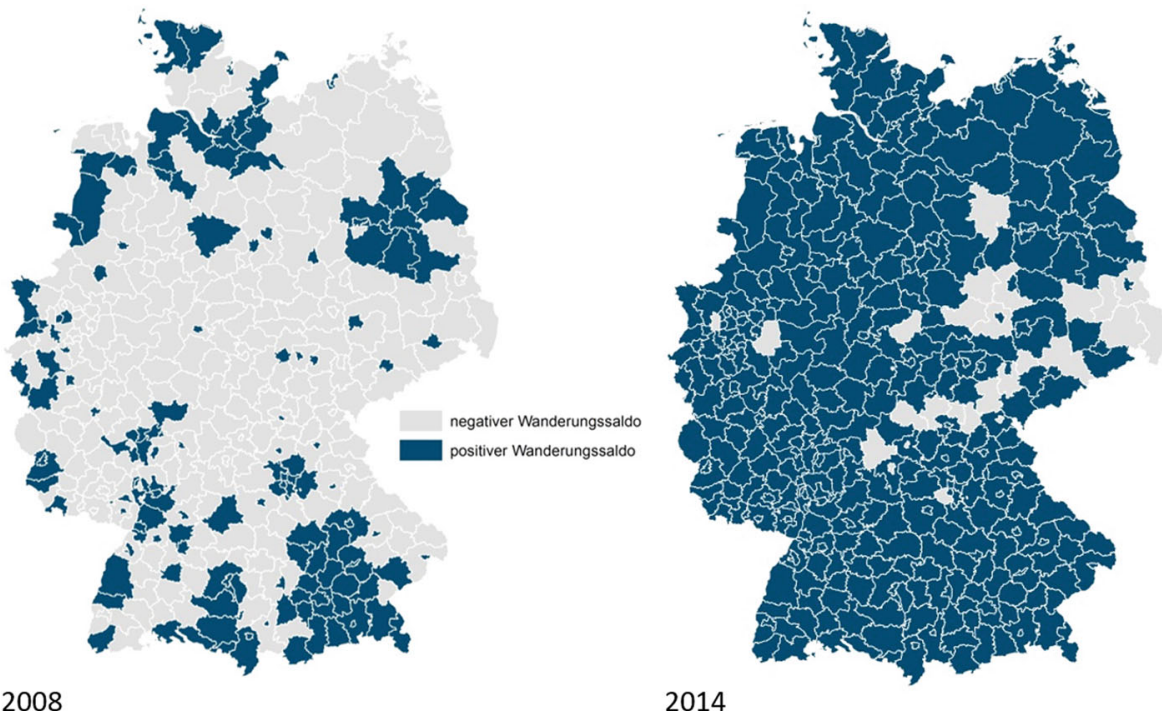

Abb. 1 Kreise und kreisfreie Städte mit positivem Wanderungssaldo (blau) im Jahr 2008 und im Jahr 2014 (Statistische Ämter des Bundes und der Länder, Wanderungsstatistik, eigene Darstellung, Kartengrundlage: Bundesamt für Kartographie und Geodäsie)

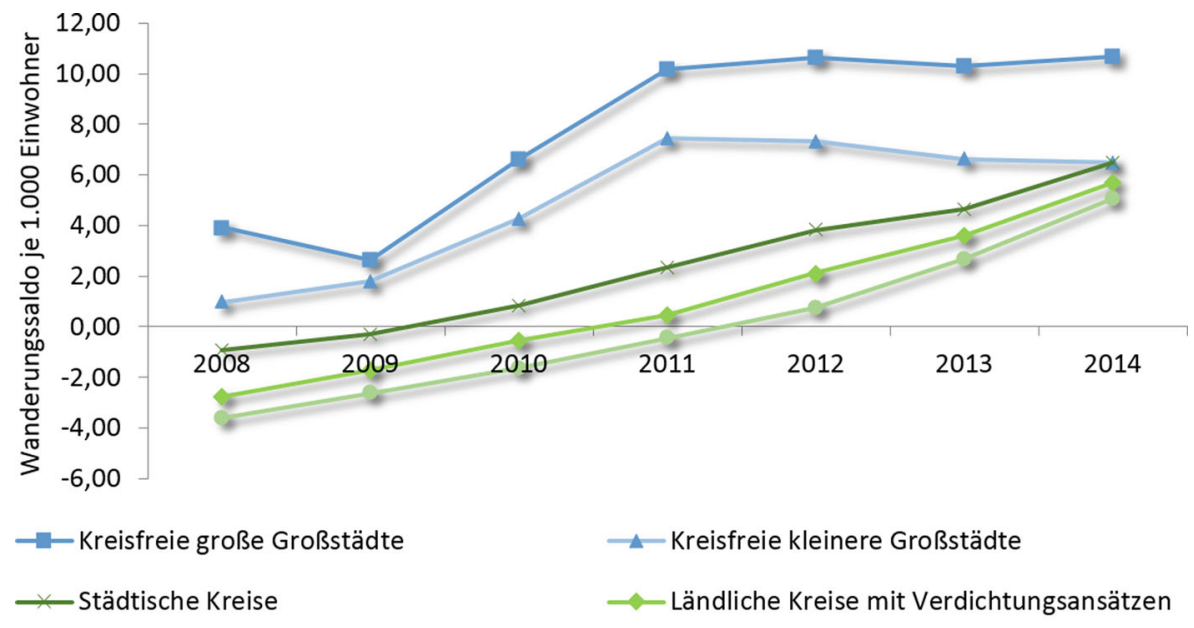

$\multimap$ Dünn besiedelte ländliche Kreise

Abb. 2 Wanderungssaldo je 1000 Einwohner differenziert nach siedlungsstrukturellem Gebietstyp (Statistische Ämter des Bundes und der Länder, Wanderungsstatistik, eigene Berechnungen und Darstellung) 


\section{Wanderungsgewinne in vielen Kommunen durch starke Zuwanderung aus dem Ausland}

Parallel dazu waren große Veränderungen bei der Außenwanderung zu beobachten. Nachdem Deutschland 2008 und 2009 noch über eine negative Außenwanderungsbilanz verfügte, kam es seit 2010 zu einem starken Anstieg der Zuwanderung aus dem Ausland. ${ }^{1}$ Insbesondere die Finanz- und Wirtschaftskrise, die zu einer hohen Arbeitslosigkeit vor allem in den südlichen Ländern Europas führte, der Beginn der Arbeitnehmerfreizügigkeit für Rumänien und Bulgarien sowie neue Krisen im Nahen Osten und anderen Teilen der Welt waren Auslöser dafür, dass der seit 2010 positive Wanderungssaldo mit dem Ausland über die Jahre immer größer wurde. Abb. 3 zeigt, dass sich seit 2009 für alle siedlungsstrukturellen Gebietstypen der Außenwanderungssaldo stark erhöht hat, wobei der Anstieg der Wanderungsgewinne mit dem Ausland in den Großstädten deutlich stärker ausgeprägt war als in den Kreisen.

Die starke Zuwanderung aus dem Ausland ist derzeit also ein entscheidender Treiber für die Entwicklung der Einwohnerzahlen und der Wanderungssaldi in den Kommunen. Da die Auslandszuwanderung jedoch aus verschiedenen Gründen insbesondere dem leichteren Arbeitsmarkteinstieg und den vorhandenen sozialen bzw. ethnischen Netzwerke in den Ballungsräumen (Henger 2014; Häußermann und Oswald 1996) - nicht auf alle Gebietstypen gleich wirkt, sondern stark auf die Groß-

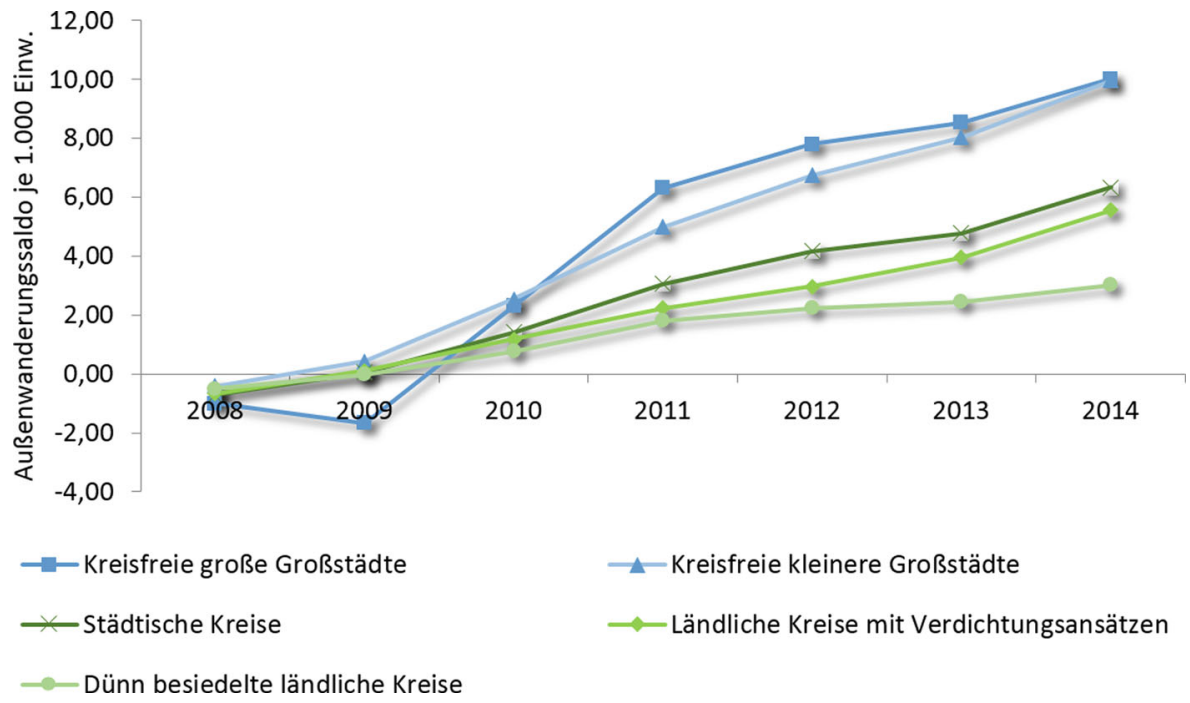

Abb. 3 Außenwanderungssaldo je 1000 Einwohner differenziert nach siedlungsstrukturellem Gebietstyp (Statistische Ämter des Bundes und der Länder, Wanderungsstatistik, eigene Berechnungen und Darstellung)

\footnotetext{
1 Bezüglich der negativen Außenwanderungssaldi in den Jahren 2008 und 2009 ist zu beachten, dass die Angaben zu den Fortzügen ins Ausland ab 2008 bis 2011 Melderegisterbereinigungen enthalten, die auf die Einführung der Vergabe von persönlichen Steueridentifikationsnummern zurückzuführen sind.
} 
städte ausgerichtet ist, beeinflusst sie derzeit in starkem Maße die innerdeutschen Wachstums- und Schrumpfungsprozesse auf kommunaler Ebene. Zudem nimmt die staatlich gesteuerte räumliche Verteilung der Asylsuchenden aktuell in großem Maße Einfluss auf die Entwicklung der kommunalen Wanderungssaldi.

\section{Fokussierung auf die innerdeutschen Wanderungen}

Die starke Auslandszuwanderung ist ein Phänomen, das sicherlich noch einige Jahre andauern wird. Die zyklische Entwicklung des Außenwanderungssaldos in der Vergangenheit lässt jedoch eine einfache Trendfortschreibung in die Zukunft als wenig sinnvoll erscheinen (Brenke und Neubecker 2013; Folkerts-Landau 2014). Vielmehr ist es wichtig, auch die sonstigen (inländischen) Wanderungstrends genau zu beobachten, um zukünftige Entwicklungen bei der räumlichen Verteilung der Bevölkerung auch für die Zeit nach Ende des aktuellen Zuwanderungszyklus abschätzen zu können. Aus diesem Grunde soll die Außenwanderung in den folgenden Untersuchungen ausgeklammert und der Fokus auf die Analyse inländischer Wanderungstrends gelegt werden.

Abb. 4 zeigt die Entwicklung des Binnenwanderungssaldos, also des Saldos der innerdeutschen Wanderungen zwischen den Kommunen, differenziert nach siedlungsstrukturellen Gebietstypen. Die Großstädte waren in den letzten Jahren die Profiteure der inländischen Wanderungen, allerdings ist ein kontinuierlicher Rückgang der Wanderungsgewinne zu beobachten, kleinere Großstädte müssen seit 2013 sogar Wanderungsverluste hinnehmen. Dies ist eine „neue“ Entwicklung, die der-

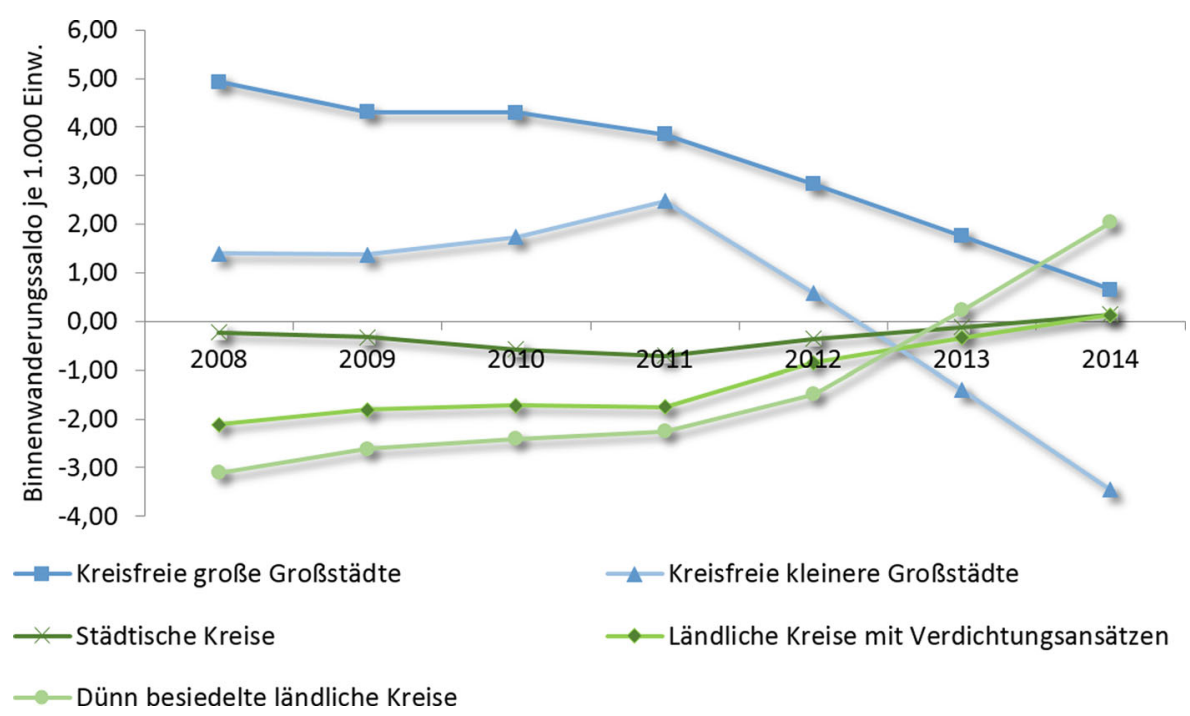

Abb. 4 Binnenwanderungssaldo je 1000 Einwohner differenziert nach siedlungsstrukturellem Gebietstyp (Statistische Ämter des Bundes und der Länder, Wanderungsstatistik, eigene Berechnungen und Darstellung) 
zeit quasi „unter dem Radar“ verläuft, weil sie durch die Auslandszuwanderung überdeckt wird.

Dem gegenüber steht jedoch ein stetig wachsender Rückgang des negativen Wanderungssaldos bei den Kreisen. Die dünn besiedelten ländlichen Kreise zeichnen sich seit 2013 sogar durch ein positives inländisches Wanderungssaldo aus und sind 2014 derjenige Gebietstyp mit dem stärksten Binnenwanderungsgewinn. Auch die städtischen Kreise und die ländlichen Kreise mit Verdichtungsansätzen weisen 2014 erstmals seit langer Zeit Binnenwanderungsgewinne aus. Bei alleiniger Betrachtung der inländischen Wanderungsbewegungen sind also ein Ende des Reurbanisierungstrends und der Beginn einer Sub- bzw. Deurbanisierungsphase festzustellen.

Die aufgezeigte Entwicklung deutet auf eine Trendumkehr hin. Wie nachhaltig ist aber die derzeit positive Einwohnerentwicklung in den einzelnen Gebietstypen und was sind ihre Treiber? Um diesen Fragen weiter nachzugehen, werden die inländischen Wanderungsbewegungen im Folgenden differenziert nach Altersklassen, Nationalität und Wanderungsdistanz untersucht.

\section{Wanderungssaldi differenziert nach Altersklassen}

Bei der nach Altersklassen differenzierten Betrachtung der Binnenwanderungen zeigt sich das bekannte und seit langem zu beobachtende Phänomen, dass Großstädte starke Wanderungsgewinne gegenüber den ländlichen Räumen bei der Altersgruppe der 18-24-Jährigen verzeichnen, also stark von der Bildungswanderung profitieren. Junge Leute ziehen aus den ländlicheren Regionen in die Großstädte, um hier eine Ausbildung zu beginnen und vor allem um hier zu studieren. Bei der Altersklasse der 25-29-Jährigen stellen vor allem berufliche Motive - der Umzug nach Antreten der ersten Arbeitsstelle - einen wichtigen Grund für Wanderungsbewegungen dar. Auch für diese Altersklasse sind die Großstädte mit ihren stark ausdifferenzierten Arbeitsmärkten sehr attraktiv (Schlömer 2009).

Die Großstädte verlieren dagegen Einwohner aus der Altersklasse der 30-49-Jährigen sowie auch der Minderjährigen (0-18-Jährigen) an die Kreise. Hauptgründe für Wanderungen von Personen aus diesen Altersklassen sind die Familiengründung und die damit verbundene Suche nach größeren, kindergerechteren Wohnflächen sowie der Erwerb von bezahlbarem Wohneigentum (Schlömer 2004). Auch von den in ihrer Dimension deutlich überschaubareren Wanderungen der ,50+“-Altersgruppen profitieren die ländlichen Räume leicht gegenüber den Großstädten und es ist entgegen der landläufigen Meinung - auch kein Attraktivitätsgewinn der Großstädte für diese Altersklasse zu beobachten. Im Gegenteil: Die Wanderungsgewinner sind auch hier die ländlichen Räume.

Beim Vergleich der altersgruppenspezifischen Wanderungssaldi im Jahr 2008 und im Jahr 2014 (siehe Abb. 5) fällt vor allem auf, dass die Verluste der Großstädte bei den Einwohnern aus der Altersklasse der 30-49-Jährigen deutlich zugenommen haben. War der Wanderungsverlust 2008 mit minus 18.518 Personen noch recht gering, so stieg er im Jahr 2014 um das 3,3fache auf minus 61.070 Personen. Die Kreise konnten die Wanderungsgewinne in dieser Altersklasse im Gegenzug deutlich steigern. 


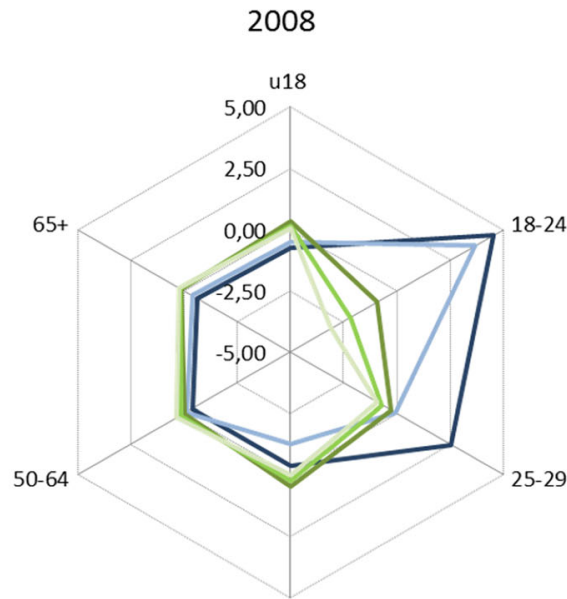

$30-49$

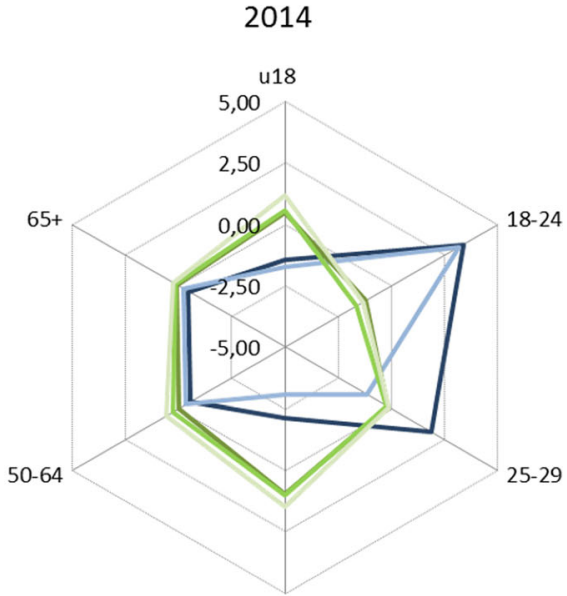

30-49
Kreisfreie große Großstädte

Städtische Kreise

-Dünn besiedelte ländliche Kreise
— Kreisfreie kleinere Großstädte

Ländliche Kreise mit Verdichtungsansätzen

Abb. 5 Binnenwanderungssaldo je 1000 Einwohner 2008 und 2014 differenziert nach Altersklassen und siedlungsstrukturellem Gebietstyp (Statistische Ämter des Bundes und der Länder, Wanderungsstatistik, eigene Berechnungen und Darstellung)

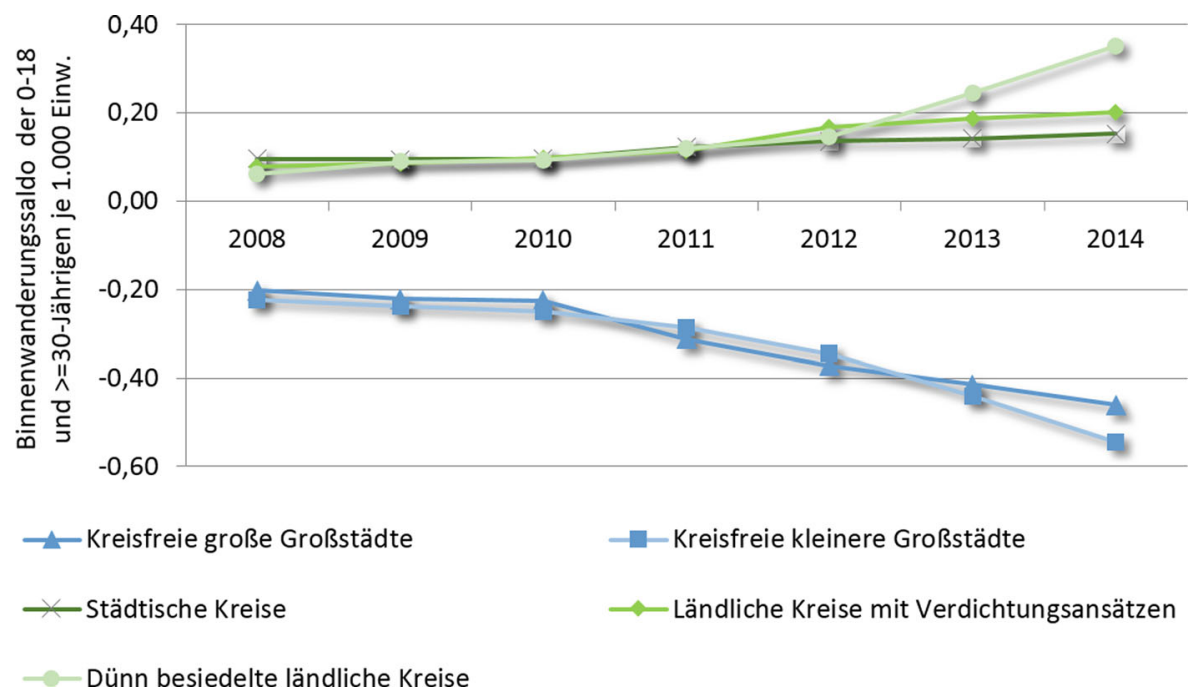

Abb. 6 Binnenwanderungssaldo je 1000 Einwohner in den Altersklassen der 0-18- und der über 29-Jährigen differenziert nach siedlungsstrukturellem Gebietstyp (Statistische Ämter des Bundes und der Länder, Wanderungsstatistik, eigene Berechnungen und Darstellung) 


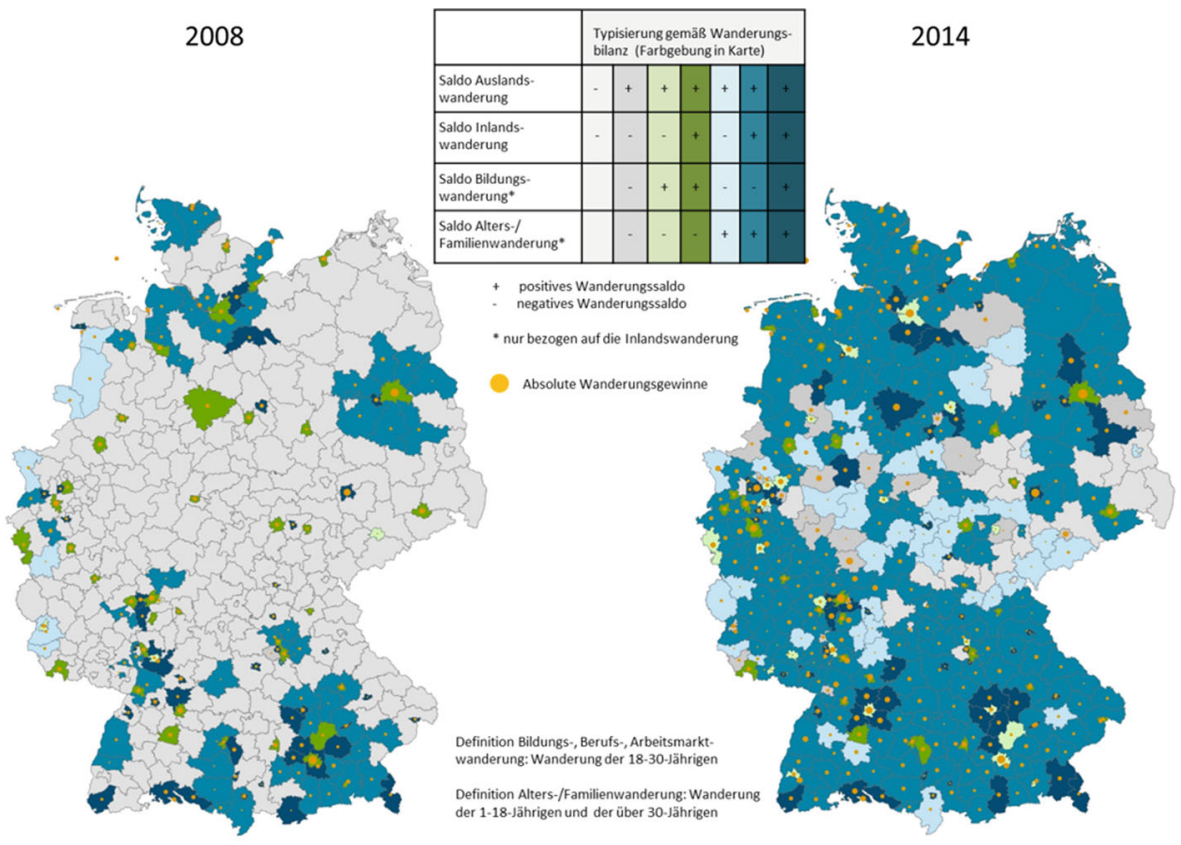

Abb. 7 Wanderungssaldi 2008 und 2014 differenziert nach altersgruppenspezifischer Wanderung, farbliche Typisierung gemäß Zuordnungstabelle (Statistische Ämter des Bundes und der Länder, Wanderungsstatistik)

In Abb. 6 sind die Binnenwanderungssaldi der „30+“-Altersgruppen inklusive der Altersgruppe der Minderjährigen zusammengefasst und die Entwicklung der Saldi für diese Altersklassen dargestellt. Hier zeigt sich, dass die Einwohnerverluste der Großstädte gegenüber den Kreisen bzw. ländlichen Kommunen seit 2008 kontinuierlich zugenommen haben. Während sich die Wanderungsverluste der großen Großstädte (>500.000 Einwohner) mehr als verdoppelt haben (von -24.459 auf -57.280), ist insbesondere bei dünn besiedelten ländlichen Kreisen ein starker Anstieg der Wanderungsgewinne um mehr als das Fünffache (von +7397 auf +41.209 ) zu verzeichnen.

Die Ausweitung der Wanderungsverluste der Großstädte bei den über 30-Jährigen führt in Städten wie München oder Hamburg dazu, dass die Wanderungsgewinne bei den 18 bis 30-Jährigen diese Wanderungsverluste nicht mehr ausgleichen können, was eine negative Binnenwanderungsbilanz zur Folge hat (siehe Abb. 7).

\section{Die Wanderungsbewegungen deutscher Staatsbürger}

Da in den letzten Jahren in größerem Umfang inländische Wanderungen festzustellen sind, die auf die Verteilung von Flüchtlingen aus Kommunen mit zentralen Erstaufnahmeeinrichtungen auf andere Kommunen zurückzuführen und damit der hier bewusst ausgeklammerten Auslandszuwanderung zuzuordnen sind, wird 

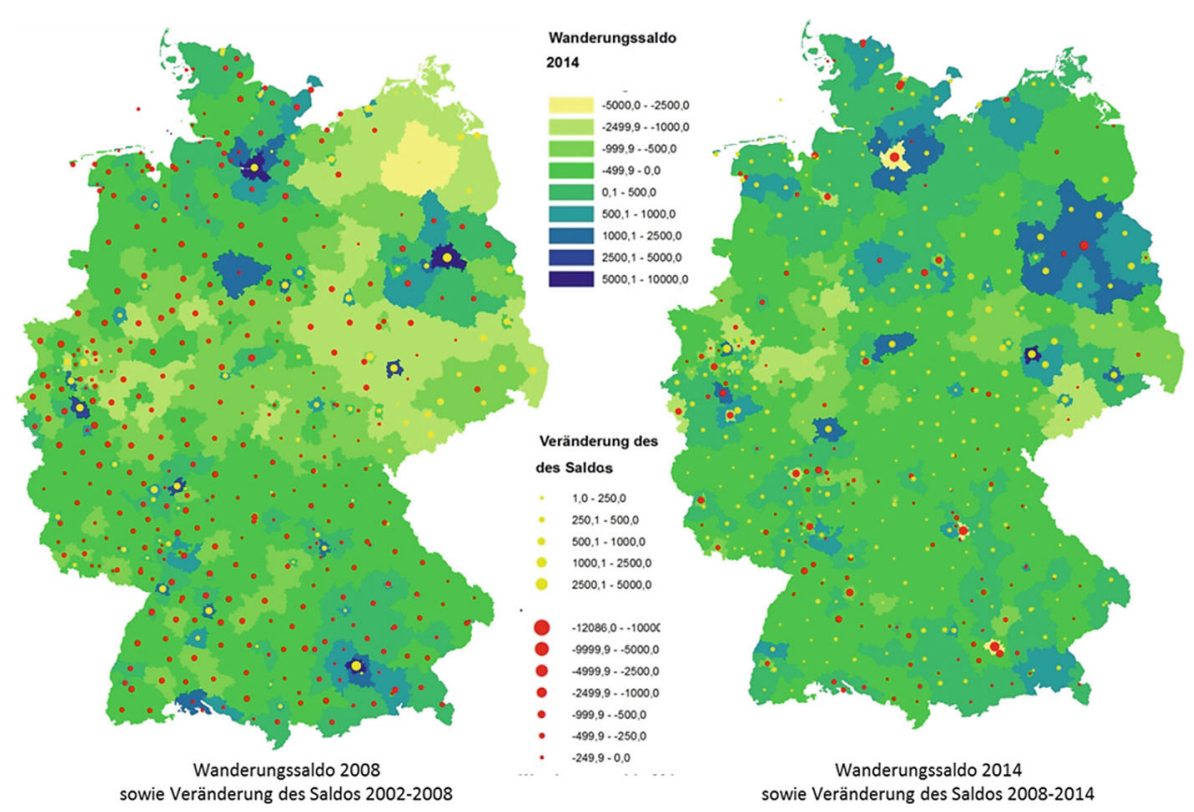

Abb. 8 Kommunale Binnenwanderungssaldi deutscher Staatsangehöriger 2008 und 2014 sowie Veränderung des Saldos 2002-2008 und 2008-2014 (Statistische Ämter des Bundes und der Länder, Wanderungsstatistik, eigene Berechnungen und Darstellung, Kartengrundlage: Bundesamt für Kartographie und Geodäsie)

im Folgenden das Binnenwanderungssaldo der deutschen Staatsangehörigen (NichtAusländer) getrennt betrachtet. Hierdurch ist es möglich, die innerdeutschen Wanderungsbewegungen der neu ins Land gekommenen Flüchtlinge auszuklammern. Allerdings werden auch die Wanderungen aller Ausländer, die bereits seit längerem in Deutschland leben, ausgeschlossen. Die kommunalen Saldi bei der inländischen Wanderung deutscher Staatsangehöriger im Jahr 2008 und im Jahr 2014 sowie die Veränderung der Saldi seit 2002 sind kartografisch in Abb. 8 dargestellt.

Bei Untersuchung der Wanderungsbewegungen der deutschen Staatsbürger zeigt sich insbesondere in den Metropolen Berlin, München und Hamburg, dass 2008 eine typische Reurbanisierungssituation gemäß dem zyklischen Stadtentwicklungsmodell von van den Berg et al. (1982, 1987, S. 88) gegeben war: Die Großstädte konnten starke Wanderungsgewinne bei Deutschen verbuchen, mit zunehmender Entfernung zur Großstadt reduzierten sich die Wanderungsgewinne und gingen in Wanderungsverluste über. Auch die meisten anderen Großstädte in Deutschland zeichneten sich 2008 durch deutliche Wanderungsgewinne in dieser Gruppe aus - lediglich in einigen Großstädten im Ruhrgebiet sowie in Ostdeutschland waren Wanderungsverluste bei den Nicht-Ausländern zu beobachten. Ebenfalls war zu dieser Zeit in fast allen peripher gelegenen ländlichen Kreisen eine negative Entwicklung des Wanderungssaldos feststellbar.

2014 zeigt sich ein komplett anderes Bild: Zwischen 2008 und 2014 hat in etlichen Großstädten ein Übergang in eine Phase der Suburbanisierung stattgefunden, der in den Städten allerdings unterschiedlich weit fortgeschritten ist. Berlin zeich- 
net sich 2014 noch durch Wanderungsgewinne deutscher Staatsangehöriger aus, diese sind seit 2008 jedoch deutlich zurückgegangen. Gleichzeitig sind die Wanderungsgewinne der Umlandkreise angestiegen und bewegen sich nun auf ähnlich hohem Niveau wie in der Hauptstadt. In Hamburg haben sich die 2008 feststellbaren Wanderungsgewinne inzwischen in Wanderungsverluste verwandelt, während die Wanderungsgewinne der Umlandgemeinden angestiegen sind. Auch in München haben sich die Wanderungsgewinne im Laufe der letzten Jahre in deutliche Wanderungsverluste verwandelt während die meisten Umlandkreise von München noch Wanderungsgewinne verzeichnen, welche aber seit 2008 zurückgegangen sind. Die negative Entwicklung der Wanderungsbilanz in Bezug auf die Nicht-Ausländer in der gesamten Stadtregion München deutet bereits auf einen Übergang in eine Phase der Deurbanisierung hin. Auch der Rückgang des negativen Wanderungssaldos in vielen ländlichen Kreisen kann als Indiz für eine beginnende Deurbanisierungsphase gesehen werden. Laut van den Berg et al. (1987) beginnt eine Phase der Deurbanisierung mit einer relativen Dezentralisierung - dies ist in der Region München derzeit zu beobachten. Allerdings ist das Bild nicht eindeutig, denn in der Region Mitteldeutschland (Sachsen, Sachsen-Anhalt und Thüringen), aber auch vereinzelt im Ruhrgebiet, finden sich weiterhin Großstädte, in denen sich die Binnenwanderungssaldi bei den deutschen Staatsangehörigen im betrachteten Zeitraum 2008-2014 zum Teil deutlich verbessert haben.

\section{Unterschiedliche Entwicklungen in den Regionen}

Die deutlichen regionalen Unterschiede hinsichtlich der Entwicklung der Binnenwanderungssaldo sind in Abb. 9 erkennbar. Diese zeigt die Entwicklung des Binnenwanderungssaldos deutscher Staatsangehöriger je 1000 Einwohner für Großstädte differenziert nach Bundesländern bzw. Regionen.

Hier wird ersichtlich, dass sich zwischen 2008 und 2014 vor allem in Nord- und Süddeutschland - und hier besonders stark in Bayern - die Bilanz der Großstädte bei der inländischen Wanderung deutscher Staatsangehöriger deutlich verschlechtert hat.

Während in den norddeutschen und bayrischen Großstädten bereits seit 2008 eine Verschlechterung des Wanderungssaldos festzustellen ist, stieg in anderen Regionen (z. B. Baden Württemberg) der positive Wanderungssaldo bis 2011 an und entwickelte sich erst seit 2012 deutlich negativ. Inzwischen sind in allen westdeutschen Regionen für die Großstädte Binnenwanderungsverluste bei den deutschen Staatsbürgern zu beobachten.

In den ostdeutschen Großstädten sind für diese Gruppe weiterhin Binnenwanderungsgewinne festzustellen, allerdings sind diese auch hier spätestens seit 2011 rückläufig. Während die Binnenwanderungsgewinne der Großstädte in der Region Berlin, Brandenburg, Mecklenburg-Vorpommern in den letzten Jahren sehr deutlich zurückgegangen sind, ist in den Großstädten der Region Mitteldeutschland nur eine langsamer Rückgang der stark ausgeprägten Wanderungsgewinne bei den deutschen Staatsbürgern festzustellen. 


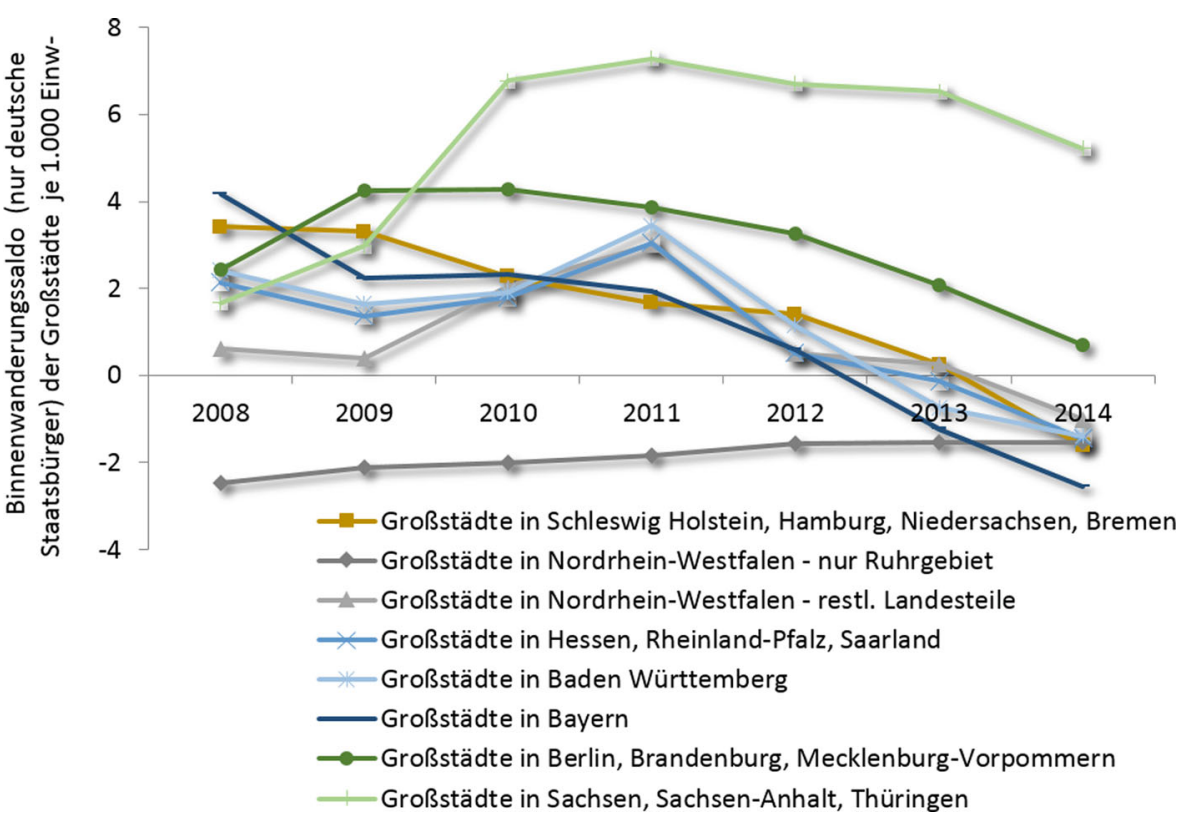

Abb. 9 Entwicklung der Binnenwanderungssaldi der Großstädte je 1000 Einwohner 2014 in verschiedenen Bundesländern bzw. Regionen bei alleiniger Betrachtung der Wanderungen deutscher Staatsangehöriger (Statistische Ämter des Bundes und der Länder, Wanderungsstatistik)

Ein anderer Trend ist in den Großstädten des Ruhrgebietes zu beobachten. Hier haben sich die Binnenwanderungsverluste bei den deutschen Staatsangehörigen seit 2008 langsam aber kontinuierlich verringert.

\section{Verdrängungsprozesse durch angespannte Immobilienmärkte in vielen Großstädten}

Als Gründe für die festgestellte Entwicklung kommen grundsätzlich sowohl Pull- als auch Push-Faktoren in Frage (Lee 1966). Ein Pull-Faktor, der die Wanderungsgewinne einiger ländlicher Kreise erklären könnte, wäre beispielsweise die Verbesserung des Bildungsangebots im ländlichen Raum durch Gründung neuer (zum Teil privater) Hochschulen (siehe z. B. MfWFT 2010). Der starke Anstieg der Immobilienpreise sowohl im Miet- als auch Eigentumsmarkt in einer Großstadt, der es Haushalten mit geringerem Einkommen und jungen Familien zunehmend schwerer macht, ihre Wohnbedürfnisse in dieser Stadt zu befriedigen, stellt auf der anderen Seite einen typischen Push-Faktor dar. Bei der räumlich differenzierten Betrachtung der Entwicklung des Binnenwanderungssaldos, aber auch bei Betrachtung der inländischen Wanderungsbewegungen deutscher Staatsbürger, sind Entwicklungen zu beobachten, die darauf hindeuten, dass vor allem Push-Faktoren für den festgestellten Trend verantwortlich sind: 


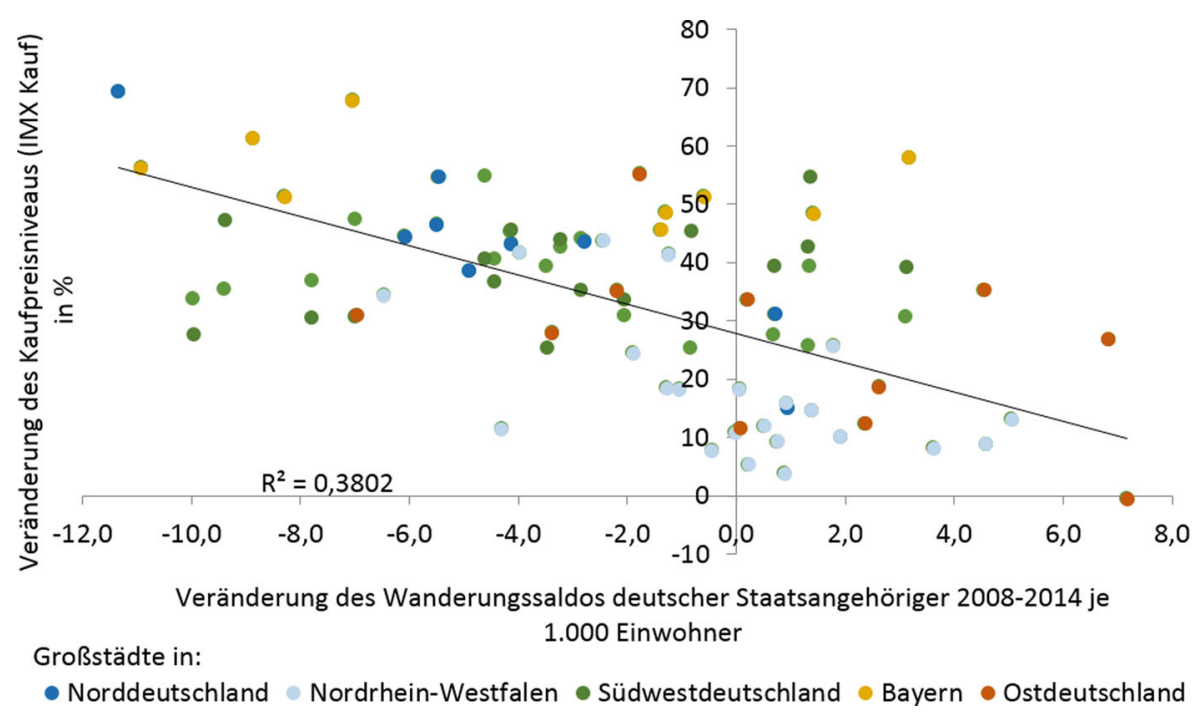

Abb. 10 Gegenüberstellung der Veränderung der Angebotspreise für Eigentumswohnungen und des Wanderungssaldos deutscher Staatsangehöriger (Statistische Ämter des Bundes und der Länder und Immobilien Scout (2015))

Auffällig ist, dass für die Großstädte in Süddeutschland sowie Berlin und Hamburg, die sich durch besonders angespannte Wohnungsmärkte auszeichnen, in den letzten Jahren eine deutlich negative Entwicklung des inländischen Wanderungssaldos festzustellen ist, während in Städten mit entspannteren Wohnungsmärkten wie den Ruhrgebietsstädten oder den Großstädten Mitteldeutschlands eine derart negative Entwicklung nicht erkennbar ist. Dies deutet auf einen Zusammenhang zwischen der Entwicklung des Binnenwanderungssaldos und der Wohnungsmarktentwicklung hin.

In Abb. 10 wird die Entwicklung der Angebotspreise für Wohnungen in den letzten fünf Jahren der Veränderung des Wanderungssaldos deutscher Staatsangehöriger zwischen 2008-2014 in den Großstädten gegenübergestellt. ${ }^{2}$ Obwohl es zahlreiche Ausreißer gibt, ist ein Zusammenhang zwischen der Entwicklung der Immobilienpreise und der Entwicklung der Wanderungssaldi erkennbar. So ist Wolfsburg nicht nur die Stadt mit dem höchsten Anstieg der Kaufpreise für Wohnungen im betrachteten Zeitraum, sondern auch diejenige mit dem stärksten Rückgang des Wanderungssaldos deutscher Staatsangehöriger. Und Chemnitz zeichnet sich in diesem Städtevergleich nicht nur als einzige Stadt in diesem Vergleich durch eine negative Wohnungspreisentwicklung aus, sondern auch durch den stärksten Anstieg des Wanderungssaldos.

Dies deutet darauf hin, dass die Wanderungsverluste der Großstädte bei der Binnenwanderung nicht auf einen generellen Attraktivitätsverlust der Städte, sondern auf

\footnotetext{
2 Bei den Daten zu den Immobilienpreisen wird auf Auswertungen der Online-Immobilienbörse Immobilien Scout zur Entwicklung der Angebotspreise für Eigentumswohnungen zurückgegriffen (Immobilien Scout 2015).
} 
einen zunehmenden Engpass an „bezahlbaren“ Wohnangeboten in den Wachstumsregionen zurückzuführen sind. In den sehr dynamisch wachsenden Städten bewegt der stark angespannte Wohnungsmarkt bestimmte Bewohnergruppen dazu, die Großstädte $\mathrm{zu}$ verlassen und sich in preiswerteren Wohnungsmarktregionen im Umland anzusiedeln.

Die Großstädte haben in den 2000er Jahren aus vielfältigen Gründen an Attraktivität gewonnen (siehe hierzu z. B. Siedentop 2008; Hesse 2010; BBSR 2012). Durch die andauernde Attraktivität und die seit einigen Jahren zu beobachtende stark auf die Großstädte ausgerichtete Auslandzuwanderung gelingt es den Städten in einer Phase der allgemeinen Bevölkerungszunahme in Deutschland jedoch immer weniger, die starke Nachfrage nach Wohnraum durch Ausweisung neuer Wohnbaufläche auf den nur sehr beschränkt zur Verfügung stehenden Grundstücken (viele militärische und infrastrukturelle Konversionsflächen sind inzwischen bebaut) zu befriedigen. Dadurch kommt ein Prozess in Gange, der gut mit Hilfe der Landnutzungstheorie von Alonso (1960) zu erklären ist. Die wachsende Nachfrage und zunehmende Nutzungskonkurrenz um die begrenzten Wohnflächen in der Stadt bewirken, dass sich die Nutzer gegenseitig für zentrale Lagen überbieten (Bietrenten) und preissensiblere Nutzer sowie Nutzer mit geringerer Zentrenaffinität ihre Wohnwünsche in den Städten nicht weiter verwirklichen können oder wollen und auf peripherere Standorte im Umland ausweichen.

Dies ist eine Entwicklung, die auch schon in der ersten Hälfte der 1990er-Jahre in Deutschland zu beobachten war. Hirschle und Schürt (2008) haben festgestellt, dass in der Zeit nach der deutschen Wiedervereinigung in dynamisch wachsenden Stadtregionen mit angespanntem Wohnungsmarkt eine deutlich ausgeprägtere Suburbanisierung festzustellen war als in Städten mit entspanntem Wohnungsmarkt. Als wichtigen Grund für die Suburbanisierung in wachsenden Stadtregionen nennen sie die große Mietpreisspanne zwischen Kernstadt um Umland (ebd., S. 223). Auch Hallenberg (2002) kommt bei der Untersuchung der Umlandwanderungen in den 1980er und 1990er-Jahren zu dem Ergebnis, dass das Wohnungsmarktgeschehen ,als primäres Erklärungsmuster für Umfang und Richtung der Umlandwanderung“ anzusehen ist (ebd., S. 138).

Zu erwähnen ist in diesem Zusammenhang, dass sich die Tendenzen der Sub- bzw. Deurbanisierung derzeit nicht nur auf die Wohnbevölkerung beschränken, sondern auch bei der Beschäftigtenentwicklung zu beobachten sind: Zwischen 2014 und 2015 ist die Zahl der Beschäftigten in den ländlich geprägten Kreisen stärker angestiegen als in den Großstädten ${ }^{3}$.

Zur genaueren Untersuchung möglicher Sub- und Deurbanisierungsprozesse werden die Wanderungen im Folgenden differenziert nach ihrer Wanderungsentfernung analysiert.

\footnotetext{
3 Veränderung der Zahl der sozialversicherungspflichtig Beschäftigtenzahlen zwischen dem 31.12.2013 und dem 30.06.2015 in unterschiedlichen Gebietstypen (Median): Dünn besiedelte ländliche Kreise $+3,03 \%$, Ländliche Kreise mit Verdichtungsansätzen $+3,25 \%$, Städtische Kreise $+2,95 \%$, Kreisfreie kleinere Großstädte $+1,75 \%$, Kreisfreie große Großstädte $+2,51 \%$ (Quelle: Bundesagentur für Arbeit, eigene Berechnung).
} 


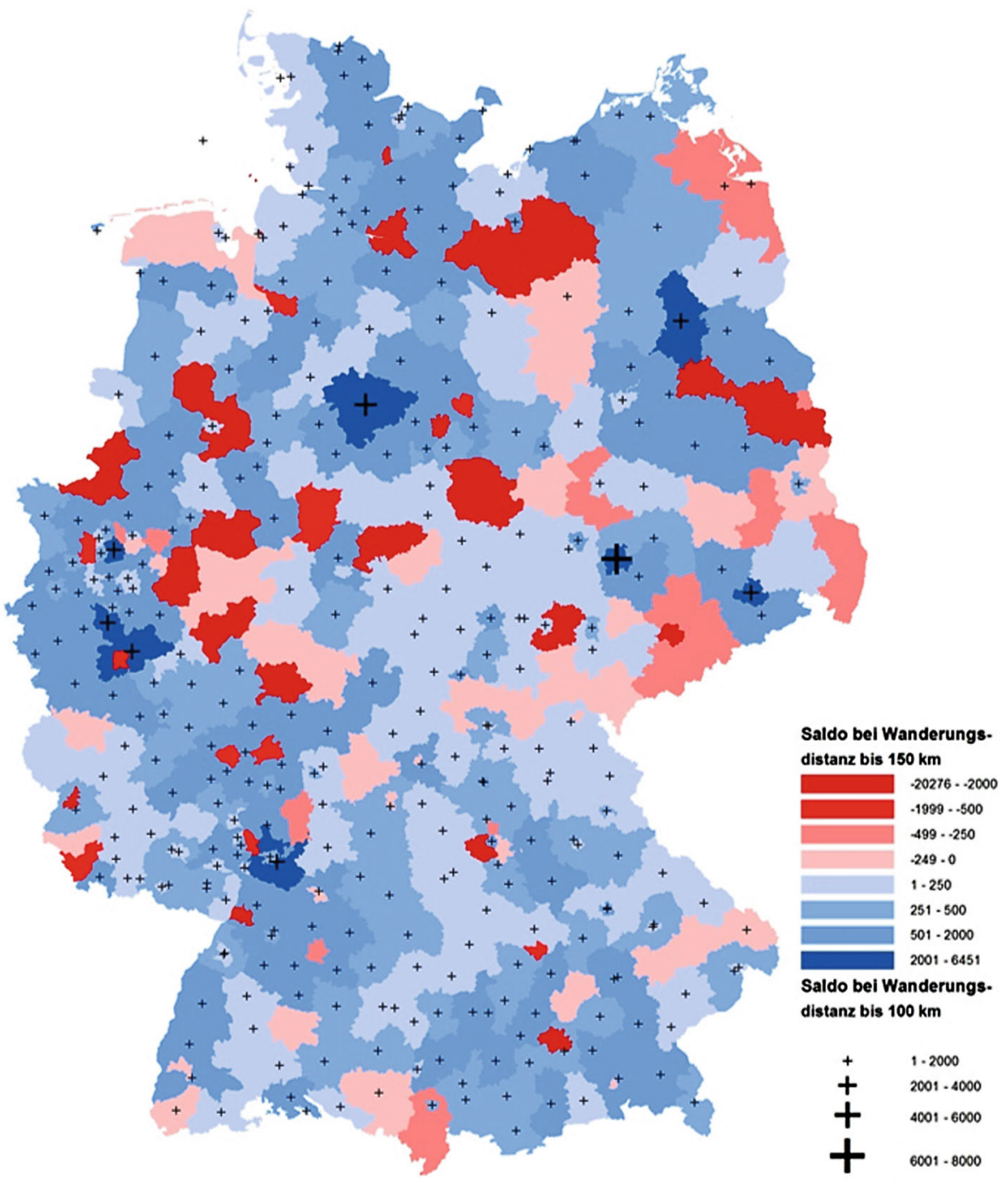

Abb. 11 Regionale Binnenwanderung 2014 (Quelle: Statistische Ämter des Bundes und der Länder, Wanderungsstatistik, eigene Berechnungen und Darstellung, Kartengrundlage: Bundesamt für Kartographie und Geodäsie)

\section{Regionale und überregionale Wanderungen}

Wenn man explizit die innerdeutschen Wanderungssaldi der regionalen Wanderungen $^{4}$ abbildet (siehe Abb. 11), zeigt sich, dass sich neben einer Vielzahl von Kreisen auch die Großstädte Leipzig und Dresden sowie mehrere Großstädte in Nordrhein-

\footnotetext{
${ }^{4}$ Regionale Wanderungen werden hier als diejenigen Wanderungen bezeichnet, die eine Entfernung von $100 \mathrm{bzw} .150 \mathrm{~km}$ nicht überschreiten.
} 
Westfalen durch Wanderungsgewinne bei der kleinräumigen, regionalen Wanderung auszeichnen. Die Großstädte dort ziehen also anders als die süddeutschen Großstädte sowie Berlin und Hamburg in zum Teil beträchtlichen Umfang Einwohner aus dem regionalen Umfeld an. ${ }^{5}$ Dies deutet auf eine weiterhin große Anziehungskraft der Großstädte hin, der aber durch die Wohnungsmarktengpässe in den strukturstarken Großstädten Grenzen gesetzt sind.

Neben den Push-Faktoren scheinen jedoch auch Pull-Faktoren eine Rolle zu spielen, worauf beispielsweise die überdurchschnittlichen Wanderungsgewinne der dünn besiedelten ländlichen Räume bei der Altersgruppe der über 30-Jährigen (und unter 18-Jährigen) hindeuten (siehe Abb. 6). Neben vielen dünn besiedelten Landkreisen im Umfeld von Großstädten (z. B. Landkreis Oberhavel oder Landkreis Lüneburg) weisen auch etliche weit von Ballungsräumen entfernt liegende Kreise wie der Landkreis Emsland positive Binnenwanderungssaldi auf.

Als interessant erweist sich hier auch die Betrachtung der innerdeutschen Fernwanderungen (siehe Abb. 12). Hier ist zunächst einmal festzustellen, dass sich $43 \%$ der Fernwanderungsgewinne ${ }^{6}$ im Jahr 2014 auf vier Städte aufteilt: die drei größten deutschen Städte Berlin, Hamburg, München sowie Leipzig. Diese Städte wirken als große „Magneten“, die aus nahezu allen Teilen des Bundesgebietes Einwohner abziehen. Daneben gehören aber auch etliche kleinere Städte und ländliche Kommunen in Nord- (insbesondere in Nordwestdeutschland) sowie in Süddeutschland zu den Gewinnern dieser innerdeutschen Fernwanderungen. Ländliche Regionen an der Küste und im Alpenvorland (z. B. Landkreis Vorpommern-Rügen, Landkreis Lörrach) profitieren dabei sicherlich über ihre landschaftlichen Reize und ihre Erholungsqualität stark von den Wanderungsbewegungen der 50+-Generation.

Dagegen sind in einer breiten Schneise, die einmal durch die Mitte Deutschlands von Ostdeutschland (mit Ausnahme des Berliner und Leipziger Umlandes sowie der Küstenregion) über Hessen bis nach Nordrhein-Westfalen, RheinlandPfalz, das Saarland sowie in Teilbereiche von Baden-Württemberg reicht, größtenteils Kommunen mit einem negativen Fernwanderungssaldo zu finden 7 . Besonders in Nordrhein-Westfalen sind starke inländische Fernwanderungsverluste festzustellen. Knapp ein Drittel (31\%) der Fernwanderungsverluste entfallen im Jahr 2014 auf NRW-Kommunen. Bei der Fernwanderung spielen neben den eher freizeit- und erholungsorientierten Gründen der 50+-Generation vor allem berufliche Gründe als Wanderungsmotive eine wichtige Rolle. Die starken überregionalen Wanderungsverluste vieler Kommunen in der Mitte Deutschlands, insbesondere der nordrheinwestfälischen Kommunen, deuten deshalb auf schwerwiegende wirtschaftsstrukturelle Probleme und einen generellen Mangel an Attraktivität dieser Regionen hin.

\footnotetext{
5 Methodisch hinzuweisen ist bei dieser Untersuchung wieder auf die Verzerrungen, die durch die Flüchtlingswanderungen aus den Erstaufnahmezentren entstehen. Für Kommunen mit Erstaufnahmeeinrichtungen wie beispielsweise dem Landkreis Gießen oder der Stadt Karlsruhe werden durch diesen ,Sondereffekt" hohe Wanderungsverluste ausgewiesen.

6 Wanderungen über mehr als 150 km (Luftlinienentfernung zwischen den Kreismittelpunkten).

7 Die starken überregionalen Wanderungsverluste des Landkreises Göttingen sind in starkem Maße auf „Sondereffekte“ durch die Erstaufnahmeeinrichtung Friedland zurückzuführen.
} 


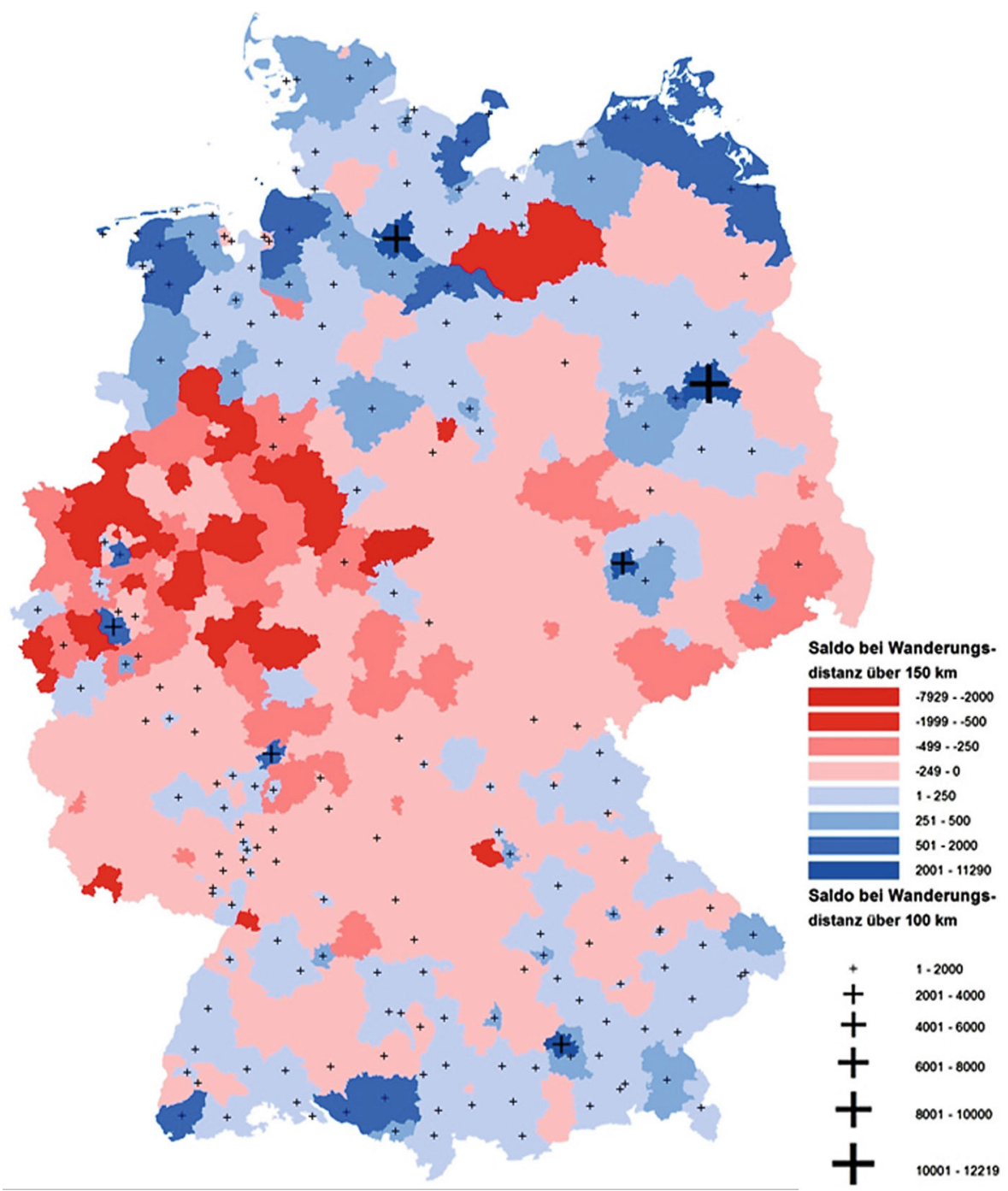

Abb. 12 Überregionale Binnenwanderung 2014 (Statistische Ämter des Bundes und der Länder, Wanderungsstatistik, eigene Berechnungen und Darstellung, Kartengrundlage: Bundesamt für Kartographie und Geodäsie)

\section{Auswirkungen auf die Raumentwicklung}

Zusammenfassend lässt sich festhalten, dass bei den inländischen Wanderungen inzwischen kein Reurbanisierungstrend mehr zu beobachten ist. In vielen Städten ist eine deutliche Verschlechterung der inländischen Wanderungsbilanz festzustellen. Etliche Städte wiesen 2014 sogar beträchtliche Wanderungsverluste auf. Für diese Großstädte besteht die Gefahr, dass der starke Anstieg der Miet- und Kaufpreise für Immobilien die wirtschaftliche Entwicklung in Zukunft negativ beeinflusst und zu 
einem nicht zu unterschätzenden Standortnachteil wird (Spars et al. 2009, S. 5 f.). Für die in den Städten ansässigen Unternehmen können die immer stärker eingeschränkten Möglichkeiten zur Verwirklichung von Wohnwünschen (unbezahlbarer Eigentumserwerb, kaum Wohnangebote in den attraktiven Lagen) zu Problemen bei der Anwerbung neuer und dem Halten der vorhandenen Mitarbeiter führen.

In den Landkreisen haben sich die inländischen Wanderungssaldi dagegen in den letzten Jahren deutlich verbessert. Neben zahlreichen Kreisen im Umland der Großstädte konnten vor allem in Nordost- sowie in Südwestdeutschland auch zahlreiche peripher gelegene ländlich geprägte Kommunen mit eigenständiger wettbewerbsfähiger Wirtschaftsstruktur oder mit besonderen landschaftlichen Reizen in den letzten Jahren eine positive inländische Wanderungsbilanz aufweisen (zum Zusammenhang zwischen Arbeitsmarkt und Fernwanderung siehe z. B. Schlömer 2009, S. 122 ff.). Daneben existieren vor allem in Ostdeutschland jedoch weiterhin etliche zumeist ländliche Kommunen, die trotz des derzeitigen Anstiegs der Gesamtbevölkerung einen Rückgang der Einwohnerzahlen zu verzeichnen haben. In vielen dieser schrumpfenden Kommunen konnte zwar in den letzten Jahren ein Rückgang des negativen Wanderungssaldos festgestellt werden, dies ist aber in vielen Fällen darauf zurückzuführen, dass große Teile der jungen, mobilen Einwohner die Kommunen bereits verlassen haben (Neu 2012).

Insgesamt ist dem durch die starke Auslandszuwanderung ausgelösten allgemeinen Bevölkerungszuwachs sowie den aktuell festzustellenden Binnenwanderungsgewinnen des ländlichen Raumes (siehe Abb. 4) allerdings eine positive Wirkung hinsichtlich des raumordnerischen Ziels der „Gleichwertigkeit der Lebensverhältnisse“ zuzuschreiben, da sich die Möglichkeiten zum Erhalt der Versorgungsinfrastruktur im ländlichen Raum durch den (zwischenzeitlichen) Rückgang der „Landflucht“ verbessert haben.

\section{Was passiert, wenn die starke Auslandszuwanderung irgendwann wieder abnimmt?}

Sollte die aktuell zu beobachtende starke Zuwanderung aus dem Ausland zukünftig zurückgehen, so ist zunächst damit zu rechnen, dass die Verdrängungsprozesse in den Großstädten abnehmen werden, was mit geringeren „Überschwappeffekten“ und einem Rückgang der positiven Wanderungssaldi in den Umlandkommunen verbunden wäre. Die derzeit zu beobachtende negative Entwicklung der inländischen Wanderungssaldi in den Großstädten scheint stark auf - vor allem immobilienmarktbedingte - Push-Faktoren und nicht auf einen Attraktivitätsverlust des großstädtischen Wohnens zurückzuführen zu sein. Besonders im suburbanen Raum ist die Nachhaltigkeit der aktuell oft sehr positiven Einwohnerentwicklung vor diesem Hintergrund dagegen zu hinterfragen.

Die bisherigen Erfahrungen zur Entwicklung der Wanderungsströme unter Schrumpfungsbedingungen (siehe z. B. Hirschle und Schürt 2008, S. 220) lassen erwarten, dass die suburbanen „Ausweichkommunen“ im Umfeld der Großstädte bei einem Ende des durch die aktuelle starke Auslandszuwanderung ausgelösten allgemeinen Bevölkerungszuwachses die Hauptleidtragenden wären. Dagegen dürf- 
ten die zurzeit aufgrund ihrer endogenen Potenziale wachsenden strukturstarken ländlichen Räume weniger stark betroffen sein. In den bereits heute schrumpfenden strukturschwachen ländlichen Räumen ist allerdings eine Fortsetzung des Prozesses der Entleerung wahrscheinlich.

Schon seit längerem hat sich zumindest bei Betrachtung der innerdeutschen Fernwanderungen das Bild eines wachsenden Nordwestens und Südostens und einer schrumpfenden Mitte Deutschlands herausgebildet (siehe z. B. Schlömer 2009, S. 82). Es deutet sich an, dass sich neben den ostdeutschen Flächenländern immer stärker auch westdeutsche Regionen - allen voran Nordrhein-Westfalen - mit dem Thema Schrumpfung intensiver als bislang auseinandersetzen müssen.

\section{Fazit}

Die Untersuchungen machen deutlich, dass die starken Wanderungsgewinne der Großstädte seit einigen Jahren in zunehmendem Maße durch die Auslandszuwanderung gespeist werden. Im Jahr 2014 sind $94 \%$ der Wanderungsgewinne der Großstädte mit mehr als 500.000 Einwohnern der Auslandszuwanderung zuzuschreiben. In vielen Großstädten sind inzwischen sogar deutliche negative Binnenwanderungssaldi feststellbar. Es konnte im Rahmen der Auswertungen gezeigt werden, dass diejenigen Großstädte, in denen die Immobilienpreise überdurchschnittlich stark angestiegen sind, besonders hohe Binnenwanderungsverluste bei den deutschen Staatsbürgern zu verzeichnen haben. Dies deutet darauf hin, dass bestimmte Haushalte die Großstädte verlassen, weil sie aufgrund der hohen Preise keine Möglichkeit sehen, ihre Wohnwünsche dort zu erfüllen. Die negative Entwicklung der Binnenwanderungssaldi der Großstädte ist demnach zumindest teilweise auf Verdrängungsprozesse aufgrund der angespannten Immobilienmärkte zurückzuführen und deutet nicht auf einen generellen Verlust der Attraktivität des großstädtischen Wohnens hin.

Interessenkonflikt R. Busch gibt an, dass kein Interessenkonflikt besteht.

Open Access Dieser Artikel wird unter der Creative Commons Namensnennung 4.0 International Lizenz (http://creativecommons.org/licenses/by/4.0/deed.de) veröffentlicht, welche die Nutzung, Vervielfältigung, Bearbeitung, Verbreitung und Wiedergabe in jeglichem Medium und Format erlaubt, sofern Sie den/die ursprünglichen Autor(en) und die Quelle ordnungsgemäß nennen, einen Link zur Creative Commons Lizenz beifügen und angeben, ob Änderungen vorgenommen wurden.

\section{Literatur}

Alonso W (1960) A theory of the urban land market. Papers and Proceedings. Pap Reg Sci Assoc 6:149-157

BBSR - Bundesinstitut für Bau-, Stadt- und Raumforschung (2010) Wohnungsmärkte im Wandel. Zentrale Ergebnisse der Wohnungsmarktprognose 2025. BBSR-Analysen KOMPAKT 01:2

BBSR - Bundesinstitut für Bau-, Stadt- und Raumforschung (2012) Die Attraktivität großer Städte: ökonomisch, demografisch, kulturell. Ergebnisse eines Ressortforschungsprojekts des Bundes. BBSR, Bonn

BBSR - Bundesinstitut für Bau-, Stadt- und Raumforschung (2015) Die Raumordnungsprognose 2035 nach dem Zensus. BBSR-Analysen KOMPAKT 05:3 
van den Berg L, Drewett R, Klaassen LH, Rossi A, Vijverberg CHT (1982) A study of growth and decline. Pergamon Press, Oxford

van den Berg L, Burns LS, Klaassen LH (1987) Spacial Cycles. Gower Publishing, Altershot

Betz R (1988) Wanderungen in peripheren ländlichen Räumen - Voraussetzungen, Abläufe und Motive. Reimer, Berlin

Birg H (2013) Soziale Auswirkungen der demographischen Entwicklung. Inf Polit Bild 2013(282):36-49

BMUB - Bundesministerium für Umwelt, Naturschutz, Bau und Reaktorsicherheit (Hrsg) (2015) Wohngeld- und Mietenbericht 2014. BMUB, Berlin

Brenke K, Neubecker N (2013) Struktur der Zuwanderungen verändert sich deutlich. DIW Wochenbericht, Bd. 49. DIW, Berlin

Deschermeier P, Müller E (2012) Analyse der Wohn- und Arbeitsortverteilung von Hochqualifizierten in der Metropolregion Rhein-Neckar. Econ Discussion Paper, Bd. 12/09. University of Mannheim/ Department of Economics, Mannheim

Dietrich-Wesbuer A, Osterhage F (2008) Wohnstandortentscheidungen in der Stadtregion: Das Beispiel „Bergisches Land“. ILS-Trends 2, Dortmund

Everett LS (1966) A theory of migration. Demography 1:47-57

Folkerts-Landau D (2014) Temporärer Zuwanderungsboom: Weckruf für die Politik? Standpunkte Deutschland. DB Research, Frankfurt am Main

Friedrich K (2008) Binnenwanderungen älterer Menschen - Chancen für Regionen im demographischen Wandel? Inf Raumentwickl 2/3:185-192

Hallenberg B (2002) Aktuelle Entwicklungen und Perspektiven der Stadt-Umland-Wanderung unter besonderer Berücksichtigung der Wohneigentumsbildung. VHW Forum Wohneigentum 3:133-142

Häußermann H, Oswald I (1996) Stadtentwicklung und Zuwanderung. In: Schäfers B, Wewer, Göttrik (Hrsg) Die Stadt in Deutschland. Aktuelle Entwicklung und Probleme. Leske + Budrich, Opladen, S 85-102

Henger R (2014) Jeder Zweite in die Großstadt. IW Kurzberichte, Köln

Herfert G, Osterhage F (2012) Wohnen in der Stadt: Gibt es eine Trendwende zur Reurbanisierung? Ein quantitativ-analytischer Ansatz. In: Brake K, Herfert G (Hrsg) Reurbanisierung - Materialität und Diskurs in Deutschland. Springer, Wiesbaden, S 86-112

Hesse M (2010) Reurbanisierung oder Metropolisierung? Entwicklungspfade, Kontexte, Interpretationsmuster zum aktuellen Wandel der Großstadtregionen. DISP 180(1):36-46

Hirschle M, Schürt A (2008) Suburbanisierung...und kein Ende in Sicht? Intraregionale Wanderungen und Wohnungsmärkte. Inf Raumentwickl 3/4:211-228

Immobilien Scout (2015) Immobilienreport 2015. Immobilien Scout, Berlin

MfWFT - Ministerium für Innovation, Wissenschaft, Forschung und Technologie des Landes NordrheinWestfalen (2010) Masterplan Studium 2020. Beste Chancen von Anfang an. MfWFT, Düsseldorf

Milbert A (2015) Wachsen oder schrumpfen? BBSR-Typisierung als Beitrag für die wissenschaftliche und politische Debatte. BBSR-Analysen KOMPAKT 12:8

Milbert A, Sturm G, Walther A (2013) Auf der Suche nach dem guten Leben. Geschlechtstypische Wanderungen in Deutschland. BBSR-Analysen KOMPAKT 04:5

Münter A (2012) Wanderungsentscheidungen von Stadt-Umland-Wanderern: regionaler Vergleich der Muster und Motive, Informations- und Wahrnehmungslücken sowie Beeinflussbarkeit der Wanderungsentscheidung in vier Stadtregionen. Monsenstein und Vannerdat, Münster

Neu M (2012) Regionale Disparitäten. Berichterstattung zur sozioökonomischen Entwicklung in Deutschland, S 185-200

Ragnitz J (2013) Wanderungen von und nach Ostdeutschland. Ifo Dresd Berichtet 6:44-48

Sander N (2014) Internal Migration in Germany, 1995-2010: New Insights into East-West Migration and Reurbanisation. Comp Popul Stud 39(2):217-245

Schlömer C (2004) Binnenwanderung seit der deutschen Einheit. Raumforsch Raumentwickl 2004(2):96-108

Schlömer C (2009) Binnenwanderung in Deutschland zwischen Konsolidierung und neuen Paradigmen. Berichte, Bd. 31. Bundesamt für Bauwesen und Raumordnung, Bonn

Siedentop S (2008) Die Rückkehr der Städte? Zur Plausibilisierung der Reurbanisierungshypothese. Inf Raumentwickl 2(3):193-210

Siedentop S, Junesch R, Klein M, Krumm R, Kleimann R (2014) Wanderungsmotive im ländlichen Raum. Ministerium für Ländlichen Raum und Verbraucherschutz Baden - Württemberg, Stuttgart

Spars G, Faller B, Jakob P, Werner AM (2009) Der Berliner Wohnungsmarkt - ein Standortvorteil. Studie im Auftrag der Investitionsbank Berlin (IBB), Wuppertal/Bonn 